\title{
Erratum: Scalar gravitational waves in the effective theory of gravity
}

\section{Emil Mottola}

Nuclear and Particle Physics, Astrophysics and Cosmology, T-2, Theoretical Division, Los Alamos National Laboratory, Mail Stop B285, P.O. Box 1663, Los Alamos, NM 87545, U.S.A.

E-mail: emil@lanl.gov

ERratum TO: JHEP07(2017)043

ARXIV EPRINT: 1606.09220

- On the last line of page 8 , the equation number referred to should be (2.9), not (2.14).

- On page 15 , sixth line, the verb form should be the plural have, not has.

- On page 20, third line above the end of section 4 contains a misspelling of the word invariant.

- On page 23, eq. (5.17) contains a misprint in its second line. Eq. (5.17) should read in its entirety:

$$
\begin{gathered}
\Pi_{i j}^{l m}(\hat{\mathbf{r}})=\frac{1}{2}\left(\delta_{i}{ }^{l} \delta_{j}{ }^{m}+\delta_{i}{ }^{m} \delta_{j}^{l}+\hat{\mathbf{r}}_{i} \hat{\mathbf{r}}_{j} \hat{\mathbf{r}}^{l} \hat{\mathbf{r}}^{m}-\eta_{i j} \eta^{l m}+\eta_{i j} \hat{\mathbf{r}}^{l} \hat{\mathbf{r}}^{m}+\hat{\mathbf{r}}_{i} \hat{\mathbf{r}}_{j} \eta^{l m}\right. \\
\left.-\delta_{i}{ }^{l} \hat{\mathbf{r}}_{j} \hat{\mathbf{r}}^{m}-\delta_{j}{ }^{l} \hat{\mathbf{r}}_{i} \hat{\mathbf{r}}^{m}-\delta_{i}{ }^{m} \hat{\mathbf{r}}_{j} \hat{\mathbf{r}}^{l}-\delta_{j}{ }^{m} \hat{\mathbf{r}}_{i} \hat{\mathbf{r}}^{l}\right)
\end{gathered}
$$

Open Access. This article is distributed under the terms of the Creative Commons Attribution License (CC-BY 4.0), which permits any use, distribution and reproduction in any medium, provided the original author(s) and source are credited. 